\title{
Monopole Antenna on Transparent Substrate and Rectifier for Energy Harvesting Applications in 5G
}

\author{
S. M. Kayser Azam ${ }^{1}$, Md. Shazzadul Islam² \\ Department of Electrical and Computer Engineering \\ International Islamic University Malaysia \\ Kuala Lumpur, Malaysia
}

\author{
A. K. M. Zakir Hossain ${ }^{3}$ \\ Fakulti Teknologi Kejuruteraan Elektrik \& Elektronik \\ Universiti Teknikal Malaysia Melaka \\ Melaka, Malaysia
}

\author{
Mohamadariff Othman ${ }^{4}$ \\ Department of Electrical Engineering \\ University of Malaya \\ Kuala Lumpur, Malaysia
}

\begin{abstract}
In line with the harvested energy required for the emerging $5 G$ technology, this article proposes a planar monopole antenna and a rectifier. The proposed Coplanar Waveguide (CPW)-fed antenna is printable on a transparent Poly-Ethylene Terephthalate (PET) substrate. The antenna has a center frequency at $3.51 \mathrm{GHz}$ within a bandwidth of $307 \mathrm{MHz}$ that covers the pioneer 5G band in Malaysia. The designed omnidirectional antenna exhibits the maximum gain of $1.51 \mathrm{dBi}$ with a total efficiency of $\mathbf{9 5 . 1 7}$ percent. At the antenna frequency, a rectifier has been designed with the voltage doubler technique on a Rogers RO3003 substrate. Over an input RF power of 0 $\mathrm{dBm}$, the rectifier has a power conversion efficiency around 42 percent. The proposed antenna rejects harmonics at least until 16 GHz frequency that makes it compatible with the rectifier to eliminate an additional bandpass filter or impedance matching network from the energy harvesting system.
\end{abstract}

Keywords-Monopole antenna; transparent substrate; 5G; energy harvesting; rectifier

\section{INTRODUCTION}

The modern communication system is moving forward to employ different applications of antennas other than only for communication purposes. For example, antennas for wearable devices, industrial and biomedical applications [1], [2] are very popular nowadays. Meanwhile, the emerging 5G technology facilitates the Internet of Things (IoT) [3] based on numerous sensor devices that require a continuous intercommunication and remote power supply at the same time. Obviously, batteries are not the only choice for empowering numerous sensor devices; power can be supplied by other sources like solar cells. In terms of remote power supply, batteries are in fact not the best choice because they need regular maintenance and replacement. Whereas, solar cells do not benefit when the sunlight is absent, or devices remain remotely indoor. On the other hand, the ambient Radio Frequency (RF) sources are always available even in the remote corners. Therefore, antennas with rectifiers [4] have drawn a special attention as energy harvesters.

In the recent times, several research works have been introduced with antennas and rectifiers for RF energy harvesting applications. Mostly for Wireless Local Area Network (WLAN) and Industrial, Scientific, and Medical (ISM) bands, RF energy harvesters have been proposed as in [5]-[8]. The RF to DC Power Conversion Efficiency (PCE) is increased by following the hierarchically similar technique of tapered matching network. In [9], a voltage doubler circuit is adopted by using Schottky diodes that are often utilized in harvesting circuits. Nevertheless, improving the PCE is a major challenge while multiple diodes are used. In [10]-[14], multilayered aperture-coupled antennas are designed and incorporated with interdigital capacitor-based voltage doubler circuits for energy harvesting applications. Some remarkable features of harmonic suppression, dual-band operation etc. are introduced through those techniques in [10]-[14]. However, the amount of input power needed to obtain a high PCE with sufficient DC output voltage in those works is not compatible for remote sensors and devices since the ambient RF power normally available in the environment is not large in amount

Antennas and rectifiers are incorporated together for energy harvesting applications [15] in frequencies other than ISM and WLAN bands. A circular loop antenna with linear polarization is utilized in [15] on an optically transparent poly-methyl methacrylate substrate at $940 \mathrm{MHz}, 1.86 \mathrm{GHz}, 2.14 \mathrm{GHz}$, and 2.49 GHz frequencies. However, designed antennas are large in dimension despite their simple structures and transparent substrates. Again, the single element shunt topology-based rectifier has limited PCEs even at the lower frequencies. Whereas, the RF power is generally captured more at low frequencies than that at high frequencies [15] because the effective area is larger at low frequencies. Also, at high frequencies, the harvested power is limited by the additional dielectric loss and the parasitic loss added by rectifying diodes. These issues can be addressed by the harmonic suppression ability [4] of antennas and rectifiers. Bandpass filters and further matching networks in typical energy harvesting systems can significantly decrease PCE and remote applicability.

To address these issues, in this article, a Planar Monopole Antenna (PMA) and a rectifier have been proposed for the pioneer 5G band in Malaysia. The antenna is designed on a transparent PET substrate that makes it suitable for 5G and IoT 
applications by allowing the sunlight to the outdoor solar cells and the wearable feature to the indoor devices. The designed antenna suppresses harmonics at least up to $16 \mathrm{GHz}$ frequency. The rectifier needs no additional impedance matching network. Therefore, the antenna with the rectifier can eliminate the need of an additional bandpass filter or impedance matching network from the energy harvester. Section II includes the antenna design, Section III demonstrates the rectifier design, Section IV discusses the results, and Section V ends the article.

\section{ANTENNA DESIGN}

In the PMA design, the CPW feeding technique has been adopted for a circular patch. In a conventional CPW-fed circular patch antenna, the radiating patch has the circular shape and the feedline is surrounded by the coplanar ground with a narrow spacing. The coplanar ground is typically large in dimension at both sides of the feedline. As a result, a high inductive path is formed on the coplanar ground. Also, the circular radiating patch is not surrounded by the ground. This results in wideband characteristics in the CPW-fed circular patch antenna. In this design, unlike the conventional circular patch CPW-fed monopole antenna, the coplanar ground is not large in size and the radiating patch is surrounded by a circular thin ground line. The design technique is illustrated in Fig. 1.

The coplanar ground has been designed in a way so that only a thin circular line is maintained as ground around the radiating patch. The antenna is symmetrically designed; thus, the circular ground line is joined to the truncated ground lines at the bottom through the thin strip-line along the feedline. The strip-line and the circular line have the same width of $w_{S}$, whereas, the strip-line has a length of $S_{L}$ at both side of the feedline that has a width of $W_{F}$ and a length of $L_{F}$. Whereas, each rectangular ground line has a width of $W_{G}$ and a length of $L_{G}$. The circular radiating patch has a radius of $r$ that is less than both the inner radius $(R)$ and outer radius $\left(R_{\text {out }}\right)$ of the circular ground line. The spacing between the radiating patch and the circular ground line is equally maintained by $R-r=$ $\Delta r$ while $R_{\text {out }}-w_{s}=R$. The PMA has a length of $L$ and a width of $W$ while the gap between the strip-line and the feedline is $g$. Dimensional parameters of the proposed antenna are provided in Table I.

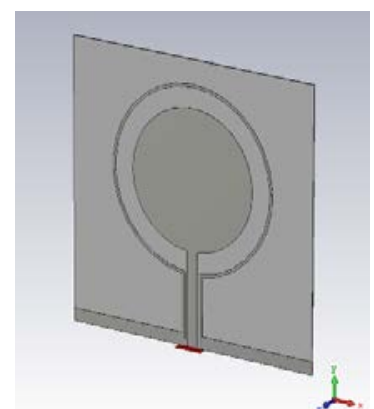

(a)

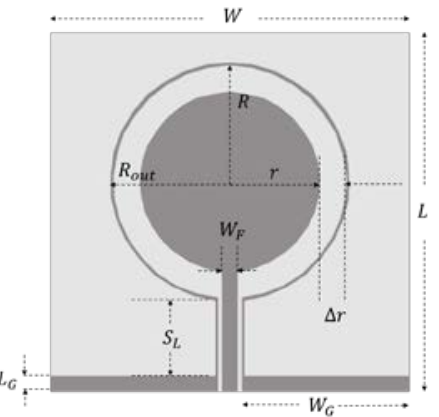

(b)
Fig 1. Proposed CPW-fed PMA (a) 3D View in Design Environment (b) Dimensional Indications.
TABLE I. PROPOSED ANTENNA DIMENSIONS

\begin{tabular}{|l|l|l|l|l|l|l|l|l|l|}
\hline Name & $\begin{array}{l}W, \\
L\end{array}$ & $\begin{array}{l}w_{s}, \\
g\end{array}$ & $r$ & $\begin{array}{l}L_{F}, \\
R_{\text {out }}\end{array}$ & $\Delta r$ & $W_{F}$ & $S_{L}$ & $L_{G}$ & $W_{G}$ \\
\hline $\begin{array}{l}\text { Unit } \\
\text { (mm) }\end{array}$ & 24 & 0.25 & 6 & 8 & 1.75 & 1 & 4.9 & 1.1 & 11.25 \\
\hline
\end{tabular}

TABLE II. PET SUBSTRATE PROPERTIES

\begin{tabular}{|l|l|l|l|}
\hline $\begin{array}{l}\text { Optical } \\
\text { Transparency } \\
(\%)\end{array}$ & $\begin{array}{l}\text { Relative } \\
\text { Permittivity, } \\
\boldsymbol{\varepsilon}_{\boldsymbol{r}}\end{array}$ & $\begin{array}{l}\text { Loss Tangent, } \\
\tan \boldsymbol{\delta}\end{array}$ & $\begin{array}{l}\text { Substrate Height, } \\
\boldsymbol{h}(\mathbf{m m})\end{array}$ \\
\hline 90.4 & 3.2 & 0.002 & 0.1 \\
\hline
\end{tabular}

The specialty of the proposed antenna is its truncated and thin coplanar ground lines surrounding the radiating patch and the feedline. It is known that conventional CPW-fed circular patch antennas have wideband characteristics. In case of the proposed antenna, the truncated and thin ground line significantly reduces the overall inductance. Such a reduced inductive characteristic results in narrowing the antenna bandwidth. At the same time, increasing the spacing between the radiating patch and the circular line of the ground decreases the overall mutual capacitance. As a result, chances of resonance at higher frequencies become less that can help the antenna to suppress harmonics. The center frequency is stably maintained by a tight coupling between the feedline and its adjacent coplanar ground lines. The antenna has been designed on an optically transparent Poly-Ethylene Terephthalate (PET) substrate by using CST Microwave Studio 2019. The designed antenna is printable and especially recommended for inkjet printing process for its fabrication. The importance of selecting the optically transparent substrate has already been discussed earlier. In addition, the low-cost PET substrates are extensively available in the market. Also, PET materials are flexible, water resistant and atmospheric-heat sustainable. Important properties of PET substrate [16] is given on following Table II.

\section{RECTIFIER DESIGN}

In conventional RF energy harvesting systems, as shown in Fig. 2(a), antennas are first connected to bandpass filters for harmonic suppression [4] so that the rectifying circuit constructed by non-linear diodes are protected from damage. Antenna with the bandpass filter is then connected to an impedance matching network for joining the rectifier. Lastly, a lowpass filter is followed by the rectifier to extract only the rectified DC power at the output terminal. However, there is a chance of power loss for the rectifier when the input RF signal pass through passive components like bandpass filters and impedance matching networks, especially at the high frequency. In fact, bandpass filters often suffer from high insertion loss [17]-[19] which is a major cause of low PCE for the rectifier. Thus, a rectifier has been proposed in this work which in and of itself can serve the purpose of bandpass filters and impedance matching networks. Hence, the proposed rectifier reduces components from the RF energy harvesting system as depicted in Fig. 2(b). 


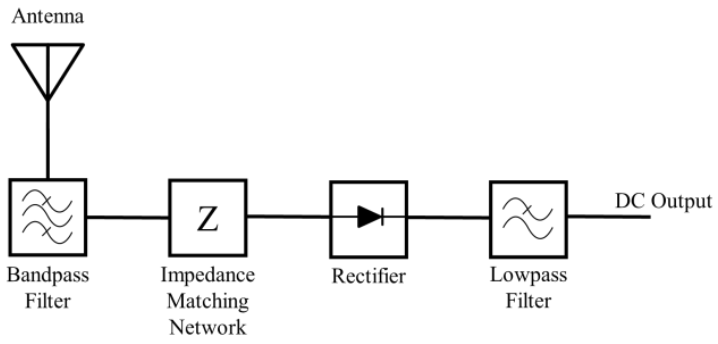

(a)

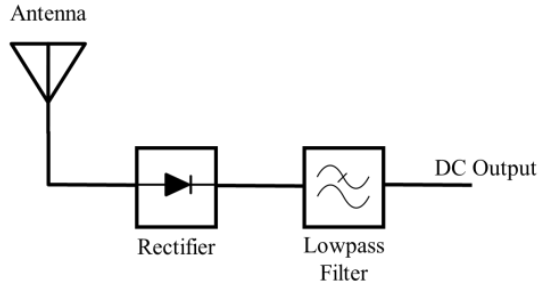

(b)

Fig 2. RF Energy Harvesting System (a) Conventional (b) Proposed.

For the proposed rectifier, two Schottky diodes (SMS7630079LF) and two capacitors (1000 pF) have been utilized on a Rogers RO3003 substrate $\left(\varepsilon_{r}=3, \tan \delta=0.001, h=0.51\right.$ $\mathrm{mm})$. Voltage doubler circuit topology has been adopted to design the rectifier circuit since this topology offers a better tradeoff between the output voltage and overall PCE than that of other rectifier topologies. The rectifier has been designed by using Advanced Design System (ADS) 2017 for both schematic and layout design. Schematic circuit, electromagnetic (EM) co-simulation set up and final layout of the proposed rectifier are demonstrated in Fig. 3.

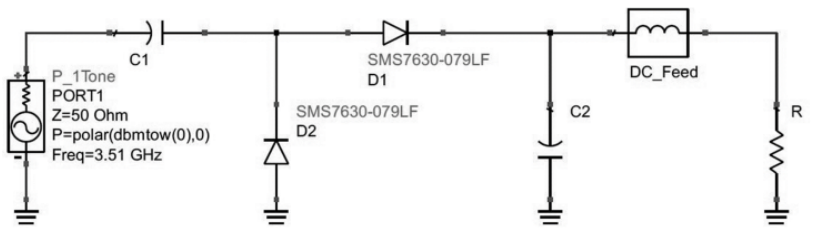

(a)

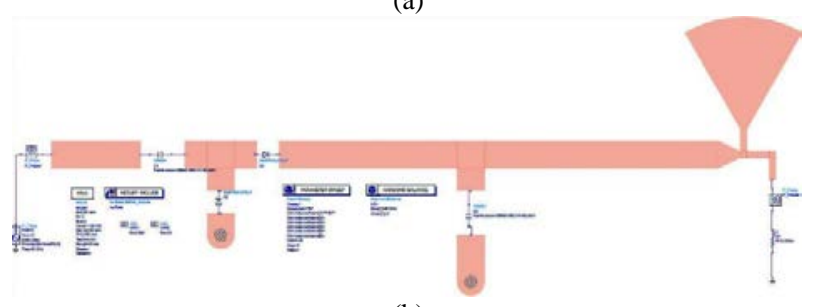

(b)

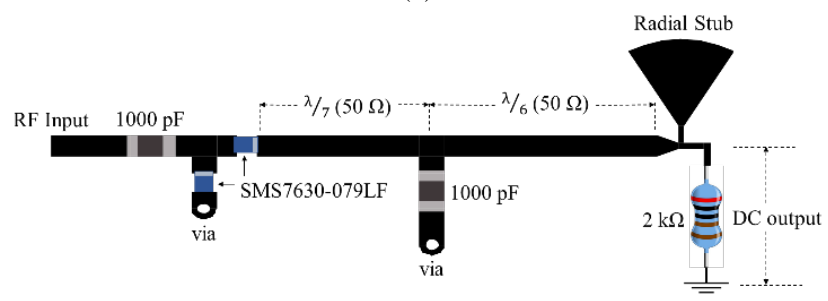

(c)

Fig 3. Proposed Rectifier Design (a) Schematic Circuit (b) EM CoSimulation Set Up (c) Final Layout.
Input terminal and core of the rectifier have been designed by employing the unequal distribution of microstrip $50 \Omega$ transmission line. The smaller microstrip $50 \Omega$ line is followed by the larger microstrip $50 \Omega$ line connected to the output terminal. In between them, the grounding capacitor is placed. Both lines are shorter than the quarter-wave line; the smaller one is constructed by a $\lambda / 7$ and the larger one is constructed by a $\lambda / 6$ length of microstrip $50 \Omega$ transmission line. This reduces the unwanted radiation loss of RF signal. At the output terminal, a tapered line has been utilized to facilitate a fine impedance matching between the $50 \Omega$ lines and high impedance $(100 \Omega)$ output lines since tapered transmission lines are useful for preventing sudden impedance mismatch [20]. From output terminal, two high impedance lines have been designed - one for connecting the output resistance and another for employing a radial stub that serves the purpose of a lowpass filter. The rectifier layout is complete after connecting a $2 \mathrm{k} \Omega$ output resistance to extract the output DC power. The overall PCE of the designed rectifier can be obtained [5] from the following equation.

$P C E=\frac{P_{D C}}{P_{R F}} \times 100(\%)$

Here, for the designed rectifier, the DC power collected from the output terminal is denoted as $P_{D C}$ while the RF power received by the antenna is represented by $P_{R F}$.

\section{RESULTS AND DISCUSSION}

The designed antenna has been simulated over a wide range of frequency from $2 \mathrm{GHz}$ to $16 \mathrm{GHz}$ by using CST Microwave Studio 2019. Impedance matching and radiation pattern of the antenna have been observed for the proposed antenna. On the other hand, ADS 2017 electromagnetic co-simulation has been performed for the final layout of the rectifier by employing the replicating models of Schottky diodes and capacitors as per the datasheet and toolkit provided by the corresponding manufacturers. Impedance matching of the rectifier has been observed to find out whether the rectifier bandwidth fits within the antenna bandwidth or not. Responses of the rectifier have been obtained analyzed in terms of its DC output voltage and PCE with respect to the change of RF input power and load resistance.

\section{A. Antenna}

One of the significant attributes of the proposed antenna is its ability to suppress the higher harmonics. S-parameter is observed to justify whether the antenna suppresses the harmonics or not. As presented in Fig. 4(a), the return loss of the antenna shows that no resonance is found below $-10 \mathrm{~dB}$ until $16 \mathrm{GHz}$ frequency. Hence, harmonic suppression has become an intrinsic characteristic of the proposed antenna which further helps the rectifier to eliminate the need of bandpass filters and impedance matching network from the RF energy harvesting system. This is one of the unique features of the proposed antenna. The minimum return loss is observed as $-39.61 \mathrm{~dB}$ at the center frequency of $3.51 \mathrm{GHz}$ with a $-10 \mathrm{~dB}$ impedance bandwidth of $307 \mathrm{MHz}$. The stability of antenna impedance matching is further justified by observing its Voltage Standing Wave Ratio (VSWR) as shown in Fig. 4(b). The minimum VSWR is found as 1.02 at the center frequency. 


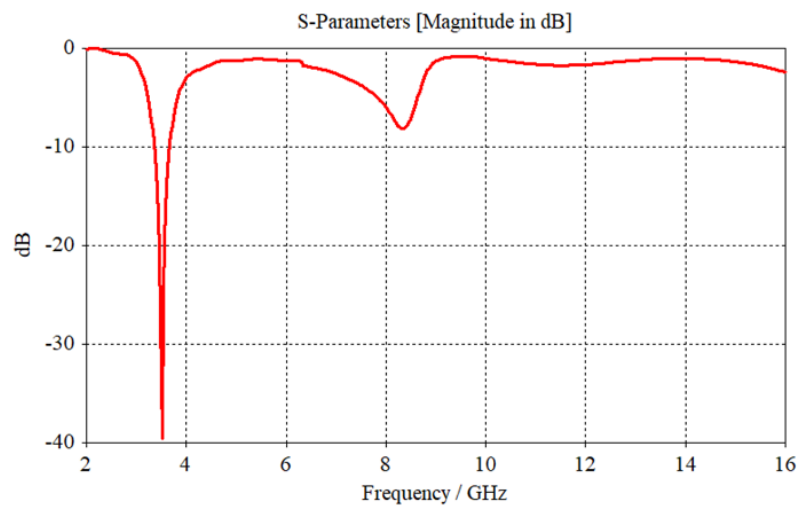

(a)

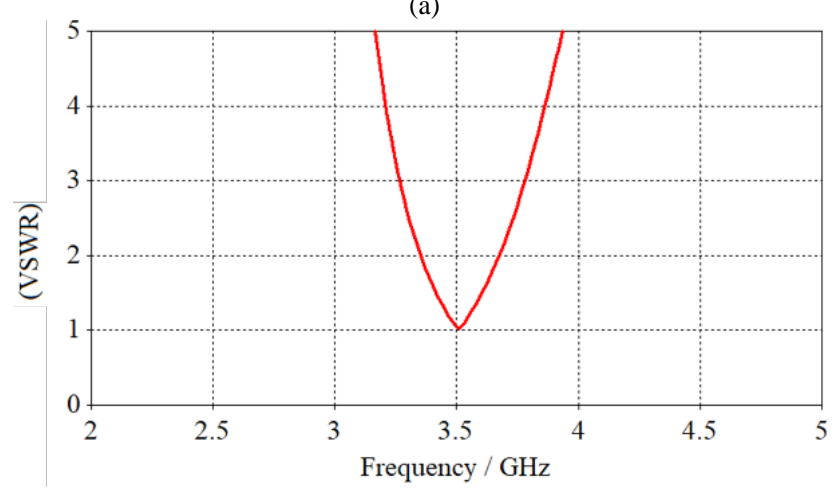

(b)

Fig 4. Impedance Matching of the Antenna (a) Return Loss (b) VSWR.

According to the antenna frequency and bandwidth, it is evident that the pioneer 5G band in Malaysia suitably fits with the designed antenna. The proposed antenna offers a maximum gain of $1.51 \mathrm{dBi}$ which is satisfactory on a transparent and flexible substrate in the $3.5 \mathrm{GHz}$ frequency range for energy harvesting applications. Since the antenna is designed by truncating a significant amount of conductive area, the antenna can exhibit such a level of gain. The designed antenna has a typical monopole omnidirectional radiation pattern that is illustrated in Fig. 5 for its center frequency.

Such an omnidirectional radiation pattern is a useful feature of the proposed antenna to collect the ambient RF energy from any direction.

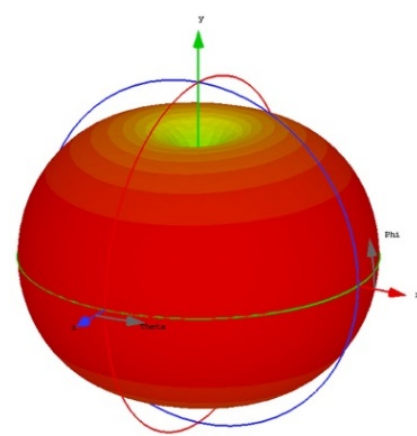

(a)

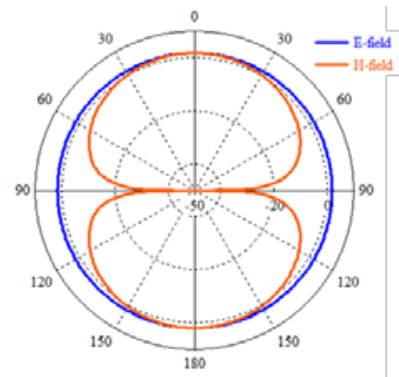

(b)
Fig 5. Radiation Pattern of the Antenna (a) 3D View (b) Polar Plot.

\section{B. Rectifier}

Return loss $\left(S_{11}\right.$ parameter) of the rectifier is presented in Fig. 6. A minimum return loss of $-23.35 \mathrm{~dB}$ is found at the rectifier center frequency of $3.51 \mathrm{GHz}$.

The $-10 \mathrm{~dB}$ impedance bandwidth of the proposed rectifier is $59 \mathrm{MHz}$ which lies within the $100 \mathrm{MHz}$ bandwidth of 5G band in Malaysia. This is an indication that the proposed antenna with the rectifier can perform a simultaneous operation of RF energy harvesting in the 5G frequency band and data communication in other bands like WiMAX.

It is common in RF rectifiers that the output DC voltage varies with the different input RF power. In Fig. 7, output response of the rectifier is presented for different input power.

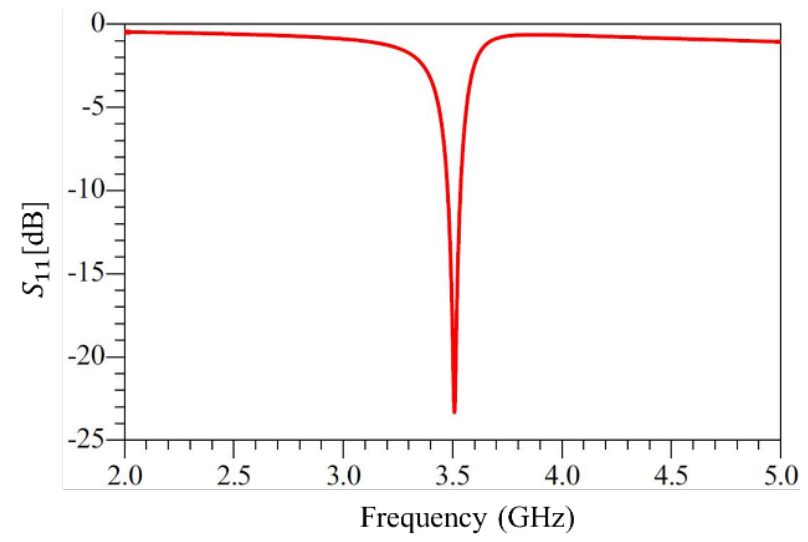

Fig 6. Return Loss of the Proposed Rectifier.

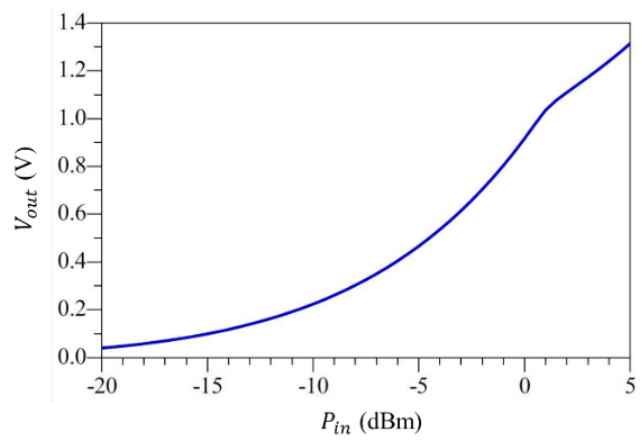

(a)

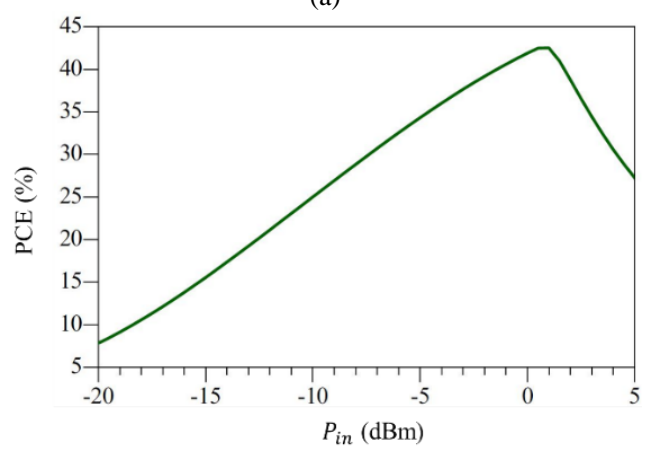

(b)

Fig 7. Rectifier Output for Different Input Power (a) $V_{\text {out }}$ (b) PCE. 


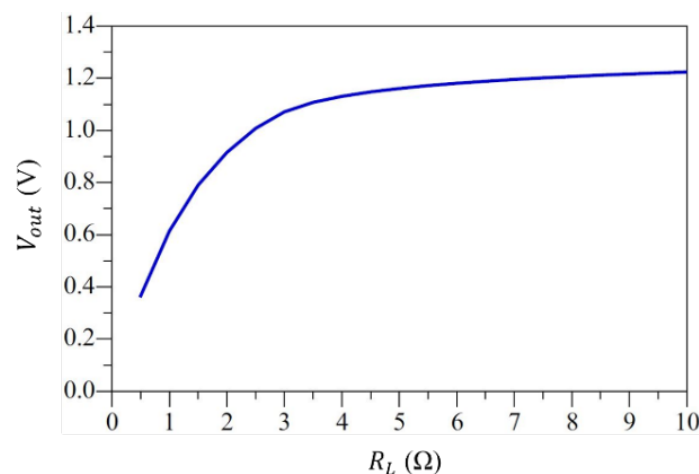

(a)

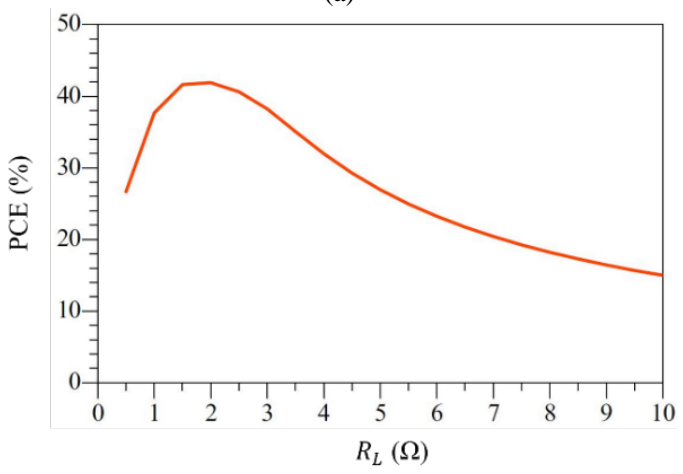

(b)

Fig 8. Rectifier Output for Different Load Resistance (a) $V_{\text {out }}$ (b) PCE.

As illustrated in Fig. 7(a), DC output voltage is $1.313 \mathrm{~V}$ when $5 \mathrm{dBm}$ input power is applied. For $0 \mathrm{dBm}$ input power, the output voltage becomes $0.915 \mathrm{~V}$. For the usual range of input RF power from $-10 \mathrm{dBm}$ to $-5 \mathrm{dBm}$, the output $\mathrm{DC}$ voltage varies from $224 \mathrm{mV}$ to $466 \mathrm{mV}$ that can drive lowpower sensor devices for the $5 \mathrm{G}$ technology. In fact, for the variation of RF input power, DC output voltage changes approximately with a linear trend within the range. This can significantly save sensor devices from damages due to sudden fluctuations in the DC voltage. As observed from Fig. 7(b), the highest PCE is found around $42 \%$ for the optimum input RF power of $0 \mathrm{dBm}$. Whereas, in case of usual range of input RF power, PCE varies from $24 \%$ to $34 \%$ which is satisfactory enough for RF energy harvesting applications in 5G frequencies.

Stability of DC output voltage due to the change of load resistance is another important aspect of the designed rectifier. Fig. 8 depicts the rectifier response with different load resistances.

Unlike many conventional rectifiers, with different load resistances, DC output voltage of the proposed rectifier is quite stable as shown in Fig. 8(a). Over a large variation of the output resistance (from $1 \mathrm{k} \Omega$ to $10 \mathrm{k} \Omega$ ), the DC output voltage changes from $0.614 \mathrm{~V}$ to $1.224 \mathrm{~V}$. This stability of DC output voltage in contrast of output resistance variation is very useful for providing a consistent amount of voltage to different nodes of a sensor network simultaneously. In Fig 8(b), variation of PCE is shown for different load resistances. With resistances from $1 \mathrm{k} \Omega$ to $10 \mathrm{k} \Omega$, it is evident that PCE does not change dramatically, rather, gradually decreases to not less than $15 \%$.
PCE is found highest (around $42 \%$ ) when $2 \mathrm{k} \Omega$ load resistance is connected at the output terminal. In terms of physical size, the rectifier is small with an area of $3.25 \times 1.25 \mathrm{~cm}^{2}$.

\section{CONCLUSION}

A printable planar monopole antenna with harmonic suppressing feature has been introduced in this work. The omnidirectional antenna has been designed on a transparent PET substrate that allows the sunlight on outdoor and flexibility on indoor conditions for the IoT and 5G applications. A voltage doubler topology-based rectifier has been proposed with the antenna for eliminating the use of bandpass filters and separate impedance matching networks in RF energy harvesting systems. The proposed antenna and the rectifier are expected to be applied on a 5G-based practical energy harvesting system in future.

\section{REFERENCES}

[1] M. S. Islam, M. I. Ibrahimy, S. M. A. Motakabber, A. K. M. Z. Hossain, and S. M. K. Azam, "Microstrip patch antenna with defected ground structure for biomedical application,” Bull. Electr. Eng. Inform., vol. 8, no. 2, pp. 586-595, 2019.

[2] M. S. Islam, M. I. Ibrahimy, S. M. A. Motakabber, and A. K. M. Z. Hossain, "A Rectangular Inset-Fed Patch Antenna with Defected Ground Structure for ISM Band,” in 2018 7th International Conference on Computer and Communication Engineering (ICCCE), 2018, pp. 104108.

[3] M. S. Islam, M. I. Ibrahimy, S. M. A. Motakabber, A. K. M. Z. Hossain, and S. M. K. Azam, "A Wideband Millimeter-Wave Printable Antenna on Flexible Substrate for Breast Cancer Imaging,” presented at the 2019 7th International Conference on Mechatronics Engineering (ICOM), Kuala Lumpur, Malaysia, Oct. 2019, [Online]. Available: https://www.researchgate.net/publication/337936173_A_Wideband_Mil limeter-

Wave_Printable_Antenna_on_Flexible_Substrate_for_Breast_Cancer_I maging.

[4] S. K. Divakaran and D. D. Krishna, "RF energy harvesting systems: An overview and design issues,” Int. J. RF Microw. Comput.-Aided Eng., vol. 29, no. 1, p. e21633, 2019.

[5] A. Eid, J. G. Hester, J. Costantine, Y. Tawk, A. H. Ramadan, and M. M. Tentzeris, "A Compact Source-Load Agnostic Flexible Rectenna Topology for IoT Devices,” IEEE Trans. Antennas Propag., vol. 68, no. 4, pp. 2621-2629, 2019.

[6] A. Eid et al., "A Flexible Compact Rectenna for $2.40 \mathrm{~Hz}$ ISM Energy Harvesting Applications,” in 2018 IEEE International Symposium on Antennas and Propagation USNC/URSI National Radio Science Meeting, Jul. 2018, pp. 1887-1888, doi: 10.1109/APUSNCURSINRSM.2018.8608525.

[7] J. Costantine, A. Eid, M. Abdallah, Y. Tawk, and A. H. Ramadan, “A load independent tapered RF harvester,” IEEE Microw. Wirel. Compon. Lett., vol. 27, no. 10, pp. 933-935, 2017.

[8] A. Eid, J. Costantine, Y. Tawk, M. Abdallah, A. H. Ramadan, and C. G. Christodoulou, "Multi-port RF energy harvester with a tapered matching network," in 2017 IEEE International Symposium on Antennas and Propagation \& USNC/URSI National Radio Science Meeting, 2017, pp. 1611-1612.

[9] M. M. Mansour and H. Kanaya, "Compact RF rectifier circuit for ambient energy harvesting,” in 2017 IEEE International Symposium on Radio-Frequency Integration Technology (RFIT), 2017, pp. 220-222.

[10] S. Ahmed, Z. Zakaria, M. N. Husain, I. M. Ibrahim, and A. Alhegazi, "Efficient feeding geometries for rectenna design at $2.45 \mathrm{GHz}$," Electron. Lett., vol. 53, no. 24, pp. 1585-1587, Oct. 2017, doi: 10.1049/el.2017.2657.

[11] S. Ahmed, Z. Zakaria, M. N. Husain, and A. Alhegazi, "Integrated rectifying circuit and antenna design with harmonic rejection for RF energy harvesting," in 2017 11th European Conference on Antennas and 
Propagation (EUCAP), Mar. 2017, pp. 1940-1944, doi: 10.23919/EuCAP.2017.7928106.

[12] S. Ahmed, Z. Zakaria, M. N. Husain, I. M. Ibrahim, N. A. Shairi, and A. Alhegazi, "High gain antenna design and doubler rectifier for microwave power transfer," in 2017 7th IEEE International Symposium on Microwave, Antenna, Propagation, and EMC Technologies (MAPE), Oct. 2017, pp. 296-299, doi: 10.1109/MAPE.2017.8250860.

[13] N. Hassan et al., "Design of dual-band microstrip patch antenna with right-angle triangular aperture slot for energy transfer application,” Int. J. RF Microw. Comput.-Aided Eng., vol. 29, no. 1, p. e21666, 2019, doi: 10.1002/mmce.21666.

[14] F. S. M. Noor, Z. Zakaria, H. Lago, and M. A. M. Said, "Dual-band aperture-coupled rectenna for radio frequency energy harvesting," Int. J. RF Microw. Comput.-Aided Eng., vol. 29, no. 1, p. e21651, 2019, doi: 10.1002/mmce.21651.

[15] S. Bellal, H. Takhedmit, and L. Cirio, "Design and experiments of transparent rectennas for wireless power harvesting," in 2016 IEEE Wireless Power Transfer Conference (WPTC), 2016, pp. 1-4.
[16] H. Dong, X. Hou, Q. Zhang, and F. Wang, "Flexible slot-ring antenna for RF wireless energy harvesting," in 2018 International Workshop on Antenna Technology (iWAT), 2018, pp. 1-4.

[17] S. M. K. Azam, M. I. Ibrahimy, S. M. A. Motakabber, and A. K. M. Z. Hossain, "A compact bandpass filter using microstrip hairpin resonator for WLAN applications," in 2018 7th International Conference on Computer and Communication Engineering (ICCCE), 2018, pp. 313316.

[18] S. M. K. Azam, M. I. Ibrahimy, S. M. A. Motakabber, A. K. M. Z. Hossain, and M. S. Islam, "A miniaturized hairpin resonator for the high selectivity of WLAN bandwidth,” Bull. Electr. Eng. Inform., vol. 8, no. 3, pp. 916-922, 2019.

[19] S. M. K. Azam, M. I. Ibrahimy, S. M. A. Motakabber, and A. K. M. Z. Hossain, "Microstrip Coupled Line Bandpass Filter with Radial Stubs for Narrow-Band Applications," Intl J GEOMATE, vol. 13, no. 40, pp. 183-188, 2017.

[20] S. M. K. Azam, M. I. Ibrahimy, S. M. A. Motakabber, and A. K. M. Z. Hossain, "Plans for Planar: Phase-Noise Reduction Techniques in Voltage-Controlled Oscillators,” IEEE Microw. Mag., vol. 20, no. 11, pp. 92-108, Nov. 2019, doi: 10.1109/MMM.2019.2935364. 